\title{
Medical education innovation: the advent of social media
}

\author{
Author: Christopher ] O’Dowd-Booth
}

\begin{abstract}
Aims
Within our day-to-day lives, social media is playing an everexpanding role. However, postgraduate training, clinical practice and research has yet to take advantage of this. With everdeveloping innovations in social technologies and access to international networks, could social education be the future of postgraduate training? This study aimed to review the current literature relating to social media in postgraduate training, and establish core trainees perceived value and role of social media as an educational tool.
\end{abstract}

\section{Methods}

A critical review of the available literature was conducted. From this a survey of postgraduate trainees was conducted, to establish the current perspective and role of social media within training. A structured questionnaire was distributed to core trainees. The results were analysed using frequency analysis and thematic analysis to develop the prevailing themes.

\section{Results}

There are limited well-designed studies directly relating to social media within training programmes. The perspective of trainees has been evaluated, with a $70 \%$ return rate from local trainees. There is a perceived value to using social networks to develop training opportunities and pursue research collaborations. There are greater opportunities for the use of social media in patient education. Trainees place a high value on social media in their training, particularly as a tool in audio-visual learning and the opportunity to develop wider networks for ongoing learning and research. However, this potentially powerful and valuable tool has not been integrated or utilised by training programmes from the perspective of the trainees surveyed.

\section{Conclusion}

The integration of social media provides an opportunity to expand both the trainee's and the patient's access to appropriate knowledge. The use of social media will enable a vast community of practice to develop and encourage an international body of

Author: Brighton and Sussex University Hospitals and University College London, UK opinion. This however is still in the early phases and requires further high quality research to establish the true impact. This study however demonstrates that trainees perceive a value and role for social media in ongoing medical education. This is an area that local and national training boards could utilise to improve training, particularly with trainees often being remote from central training locations. To improve care with limited resources, adoption of new technology is essential, and with the ever-increasing role of social media outside of medicine, this should not be overlooked.

\section{Conflict of interest statement}

None to declare. 